\title{
Calcium reduced PMS symptoms during the luteal phase of the menstrual cycle
}

\author{
Thys-Jacobs S, Starkey P,Bernstein D, et al, and the Premenstrual Syndrome Study Group. Calcium carbonate and the premenstrual \\ syndrome: effects on premenstrual and menstrual symptoms. Am J Obstet Gynecol 1998 Aug;179:444-52.
}

\section{Question}

Can calcium supplementation reduce the symptoms that occur during the luteal and menstrual phases of the menstrual cycle in women with premenstrual syndrome (PMS)?

\section{Design}

Randomised, double blind, placebo controlled trial.

\section{Setting}

12 outpatient health centres in the US.

\section{Participants}

497 healthy, premenopausal women aged 18-45 years (mean age 33 y, 80\% white) who had regular menstrual cycles, a history of cyclically recurring PMS, PMS symptoms of at least moderate severity confirmed by a validated PMS diary for $\geqslant 2$ menstrual cycles, and $\geqslant 1$ of the following: mood swings, depression, tension, anxiety, anger, or crying spells. Exclusion criteria were use of analgesics, digitalis, bile acid resin binders, or levonorgestrel; chronic or excessive use of calcium based antacids or calcium supplements; renal disease or colic; hypoparathyroidism or primary hyperparathyroidism; gastrointestinal or hepatic disease; inflammatory bowel disease or malabsorption; mental illness; gynaecological abnormality; pregnancy or breast feeding; unwillingness to use contraception; or contraceptive use $<6$ months before enrolment.

\section{Intervention}

Women were allocated to receive elemental calcium, 1200 $\mathrm{mg} /$ day, in the form of calcium carbonate $(\mathrm{n}=248)$ or placebo $(n=249)$, and instructed to begin treatment 7 to 10 days after cessation of menses and to continue treatment for 3 menstrual cycles.

\section{Main outcome measures}

Mean symptom complex scores (a mean of 17 core PMS symptom ratings) and symptom factor scores (ratings of 4 symptom factors: negative affect, water retention, food cravings, and pain) measured by a daily self assessment questionnaire (the PMS diary) during the luteal, menstrual, and intermenstrual phases of the menstrual cycle.

\begin{abstract}
Main results
Compared with baseline, the calcium treated group had lower mean symptom complex scores during the luteal phase of the second $(\mathrm{p}<0.05)$ and third $(\mathrm{p}<0.001)$ treatment cycles. By the third treatment cycle, the mean luteal phase symptom complex score was reduced from baseline by $48 \%$ in the calcium treated group and $30 \%$ in the placebo group. By the third treatment cycle, the calcium treated group had lower scores for all 4 symptom factors compared with baseline and placebo during the luteal phase (both $\mathrm{p}<0.05$ ); negative affect was reduced by $45 \%$ for calcium compared with $28 \%$ for placebo, water retention was reduced by $36 \%$ for calcium compared with $24 \%$ for placebo, food cravings were reduced by $54 \%$ for calcium compared with $34 \%$ for placebo, and pain was reduced by $54 \%$ for calcium but increased by $15 \%$ for placebo. These effects were not shown for the menstrual or intermenstrual phases of the menstrual cycle.
\end{abstract}

\section{Conclusion}

After 3 menstrual cycles, calcium supplementation reduced core premenstrual syndrome symptoms and negative affect, water retention, food cravings, and pain during the luteal phase of the menstrual cycle in women with premenstrual syndrome.

Source of funding: SmithKline Beecham Consumer Healthcare.

For correspondence: Dr S Thys-Jacobs, St Luke's-Roosevelt Hospital Center, 425 West 59 th Street, Suite 9C, New York, NY 10019, USA. Fax +1 2125238102.

\section{Commentary}

Concerned about a possible "quick fix" approach to the treatment of PMS, I read the study by Thys-Jacobs $e t$ al with caution, especially because it was funded by a drug company. This large experimental study, however, is well designed and provides convincing evidence that calcium supplementation reduces PMS symptoms. Calcium supplements for relief of the physical and emotional symptoms that plague women in their menstrual years, appear to be an attractive alternative to current medicinal treatments. Prescribing gonadotrophin releasing hormone agonists and serotonin reuptake inhibitors (eg, Prozac) with their documented side effects, perpetuates the medicalising of bodily functions that should be considered within the normal range of women's experience.
During the past 50 years, many medicinal and non-medicinal treatments have been suggested for PMS including diet (limit salt, sweet foods, and caffeine); vitamin supplements (vitamin $\mathrm{B}_{6}$ and evening primrose oil); lifestyle changes (regular exercise, and decreased smoking and alcohol consumption); and alternative healing modalities (herbal therapies such as bach flower remedies and raspberry leaf tea [which interestingly is high in calcium], acupuncture, and aromatherapy). Anecdotal evidence and descriptive and small scale experimental studies support many of these treatments, ${ }^{12}$ but their efficacy is scientifically disputed. Although, as ThysJacobs et al note, questions remain about the required dosage of calcium carbonate and duration of treatment, calcium supplementation in the dosage tested (1200 $\mathrm{mg} / \mathrm{d}$ ) appears to be a relatively safe thera- peutic option for treatment of luteal phase premenstrual symptoms (from ovulation to the onset of menstruation). It also has the added benefit of increasing the intake of calcium and therefore contributes to the prevention of osteoporosis. As women predominate in nursing and midwifery, the topic of effective treatment of PMS is personally and professionally pertinent. Calcium supplementation certainly adds to the available PMS therapeutic repertoire.

Lynne S Giddings, RGON, RM, PhD Principal Lecturer School of Nursing and Midwifery Auckland Institute of Technology Auckland, New Zealand

1 Stewart M. Beat PMS through diet. London: Vermilion, 1994.

2 Lilly C. Why am I such a bitch? New Zealand: Hodder Moa Beckett, 1997. 